\title{
Extensive beam test study of prototype MRPCs for the T0 detector at the CSR external-target experiment
}

\author{
Dongdong Hu ${ }^{1,2,3, a}$, Jiaming Lu ${ }^{1,2}$, Jian Zhou ${ }^{1,2}$, Peipei Deng ${ }^{1,2}$, Ming Shao ${ }^{1,2, b}$, Yongjie Sun ${ }^{1,2, c}$, Lei Zhao ${ }^{1,2, d}$, \\ Hongfang Chen ${ }^{1,2}$, Cheng $\mathbf{L i}^{1,2}$, Zebo Tang ${ }^{1,2}$, Yifei Zhang ${ }^{1,2}$, Yi Zhou ${ }^{1,2}$, Wenhao You ${ }^{1,2}$, Guofeng Song ${ }^{1,2}$, \\ Yitao $\mathrm{Wu}^{1,2}$, Bing $\mathrm{Zhu}^{2}$ \\ ${ }^{1}$ State Key Laboratory of Particle Detection and Electronics, University of Science and Technology of China, Hefei 230026, \\ People's Republic of China \\ ${ }^{2}$ Department of Modern Physics, University of Science and Technology of China, Hefei 230026, People's Republic of China \\ ${ }^{3}$ University of Science and Technology of China, No. 96, JinZhai Road Baohe District, Hefei 230026, Anhui, People's Republic of China
}

Received: 26 September 2019 / Accepted: 5 March 2020 / Published online: 28 March 2020

(C) The Author(s) 2020

\begin{abstract}
The Cooling Storage Ring (CSR) External-target Experiment (CEE) will be the first large-scale nuclear physics experimental device at the CSR of the Heavy-Ion Research Facility in Lanzhou (HIRFL) in China. A new T0 detector has been proposed to measure the multiplicity, angular distribution and timing information of charged particles produced in heavy-ion collisions at the target region. Multi-gap resistive plate chamber (MRPC) technology was chosen as part of the construction of the $\mathrm{T} 0$ detector, which provides precision event collision times (T0) and collision geometry information. The prototype was tested with hadron and heavy-ion beams to study its performance. By comparing the experimental results with a Monte Carlo simulation, the time resolution of the MRPCs are found to be better than $\sim 50 \mathrm{ps}$. The timing performance of the $\mathrm{T} 0$ detector, including both detector and readout electronics, is found to fulfil the requirements of the CEE.
\end{abstract}

\section{Introduction}

One of the main purposes of heavy-ion collisions is to study the bulk properties of strongly interacting matter and to understand the quantum chromo dynamics (QCD) phase diagram [1]. At finite temperature (T) and chemical potential $(\mu)$, QCD describes relevant features of nuclear physics in the early universe, in neutron stars and in heavy ion collisions. By varying the collision energy, different nuclear mat-

\footnotetext{
a e-mail: hurongr@mail.ustc.edu.cn (corresponding author)

be-mail: swing@ustc.edu.cn

c e-mail: sunday@ustc.edu.cn

de-mail: zlei@ustc.edu.cn
}

ter states and phase structure can be explored. In the region of low temperature and high net baryon density, the nuclear equation of state (EOS) is most important for understanding the phase diagram, gaining a better insight of the properties of stellar objects and heavy nuclei [2-4], and confirming the possible occurrence of a hypothetical quarkyonic matter phase at very high baryon number density [5-10]. More theoretical and experimental efforts are definitely required to arrive at a convincing constraint of $\mathrm{E}_{\text {sym }}(\rho)$ [11]. Dedicated heavy-ion experiments at energies of several hundred $\mathrm{AMeV}$ will help resolve these large theoretical uncertainties, which is well covered by the CSR of the Heavy-Ion Research Facility in Lanzhou (HIRFL).

The CSR external-target experiment (CEE) has been proposed to study (1) the density dependence of nuclear symmetry energy by measuring the $\pi^{-} / \pi^{+}$ratio (and other relevant observables) for various heavy-ion collision systems, (2) the EOS at supra-saturation density, and (3) the rich QCD phase at high-density and low-temperature. The CSR [12] can deliver a wide range of heavy-ion beams from deuteron (up to $1 \mathrm{AGeV}$ ) to uranium nuclei (up to $520 \mathrm{AMeV}$ ), and therefore, can provide significant opportunity to study $\mathrm{E}_{s y m}(\rho)$ and the properties of cold nuclear matter and quarkyonic matter phase at very high baryon number density. For example, the $\pi^{-} / \pi^{+}$ratio in heavy-ion collision in this energy region can be a sensitive probe [11].

The CEE system includes a large angle dipole magnet, tracking detectors, a Time of Flight (TOF) system, and a zero-degree calorimeter (ZDC), as depicted in Fig. 1 [13]. The TOF system contains a T0 detector, an internal TOF (iTOF) and an external TOF (eTOF). The T0 detector is located at a $\sim 10$ centimeter distance around the target region to detect the final-state charged particles and clusters in the 


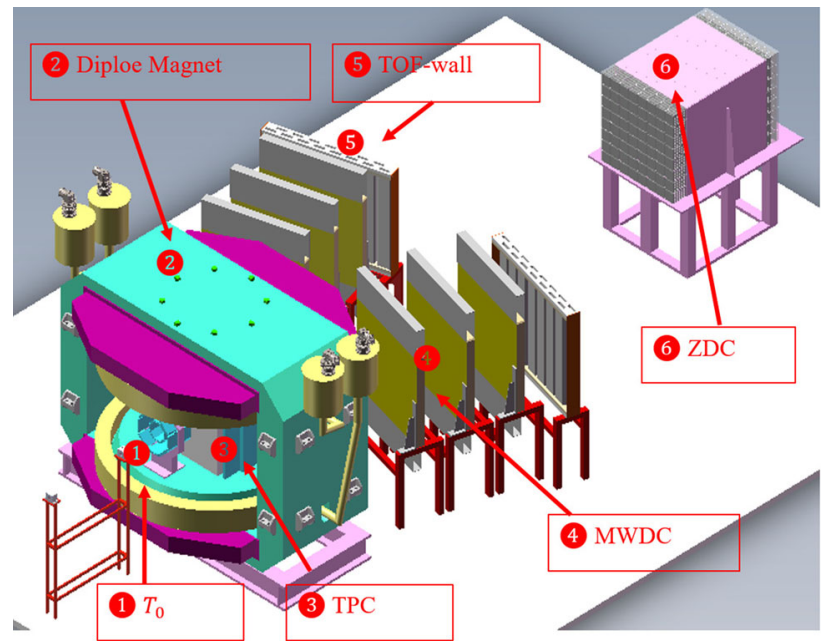

Fig. 1 Schematic layout of the CEE detector system, taken from Ref. [13]

heavy-ion reaction. Both the target and the $\mathrm{T} 0$ detector sit in a strong magnetic field (maximal 0.5 Tesla). The TOF technique is employed to identify the charged final-state particles. As the start detector of the TOF system, the T0 detector not only determines the collision time with high precision but also serves as a trigger detector for the experimental system, providing information on the event multiplicity and reaction plane.

In the GEANT4 simulation, a kinetic energy 1.0 AGeV Ar-Ar collisions were generated by UrQMD3.4.6 [14], and $\sim 10,000$ UrQMD data generated events were fed to the MC detector system to test the TOF performance. The time difference between the collision point and the hit on TOF detector was recorded. TOF timing uncertainties of 50, 100, 150 and $200 \mathrm{ps}$ were studied. A 5\% smearing to the particle momentum was added to account for the reconstruction uncertainty. Track length uncertainties of 0.5 and $2 \mathrm{~cm}$ were also included in the simulation for particles hitting the iTOF and eTOF, respectively (we will write a paper to illustrate the entire simulation processs.). The simulation results are shown in Fig. 2 , for both the iTOF and eTOF, with the TOF time resolution setting at $100 \mathrm{ps}$. In the plots, the red and blue areas denote bands within $2 \sigma$ of the $\mathrm{m}^{2}$ distribution of pions and protons as a function of momenta. The pink arrow marks the upper momentum under which $99.5 \%$ of the pions reside. The black arrow has a similar meaning for protons. There were very few kaon in the final state, so they were neglected in the plots. It is clear from the figure that the pion/proton separation is easier for eTOF because of the much longer flight path $(>2.5 \mathrm{~m})$ than iTOF $(0.5-1.2 \mathrm{~m})$, and correspondingly longer minimal time-of-flights for a eTOF $(\sim 8 \mathrm{~ns})$ than iTOF $(\sim 2.5 \mathrm{~ns})$. A TOF system with an overall time resolution of 100ps is quite adequate for pion/proton identification for the CEE.

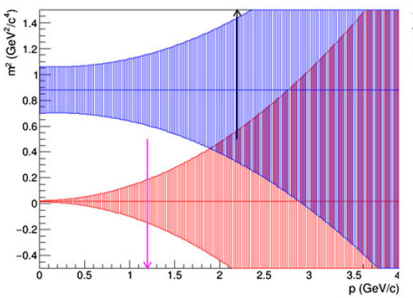

(a)

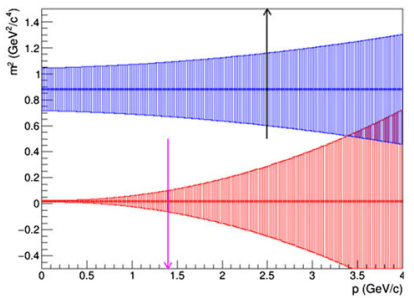

(b)
Fig. $2 \mathrm{~m}^{2}$ distribution vs. particle momentum, measured by the CEE iTOF (a) and eTOF (b). In both plots, the red and blue areas identify bands within $2 \sigma$ of the $m^{2}$ distribution of pions and protons as a function of momenta. The pink arrow marks the upper momentum under which $99.5 \%$ of the pions reside. The black arrow has a similar meaning, but for the protons

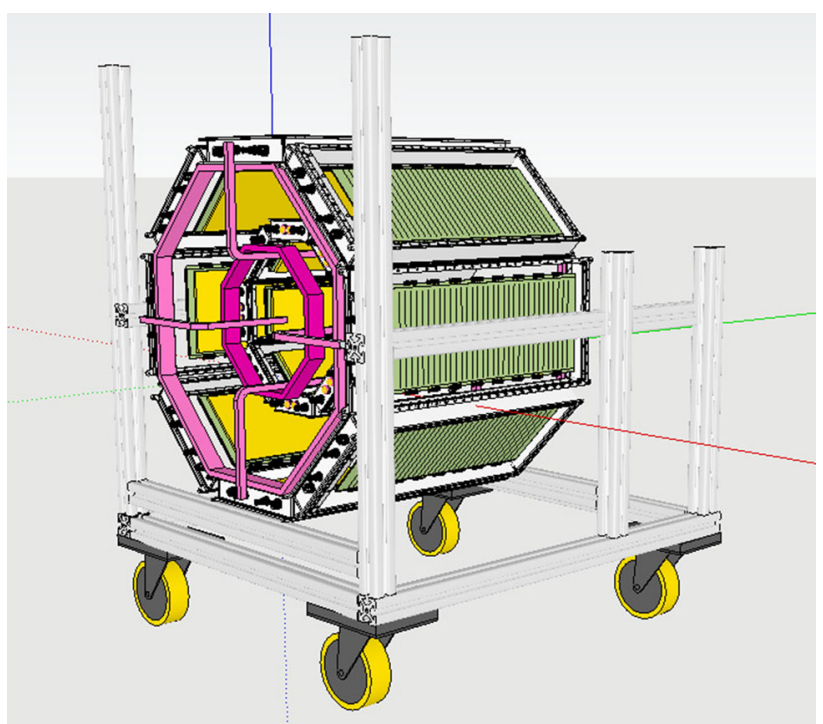

Fig. 3 Sketch of the structure of T0 detector, which is composes of inner and outer layers. Each layer includes eight MRPCs

It's noted that in Fig. 2 the 100 ps time resolution includes intrinsic contribution from both T0 detector and iTOF and eTOF detectors. For example, a 70 ps time resolution for both the T0 and iTOF detector combine to roughly $100 \mathrm{ps.}$ In our design the intrinsic time resolution for the $\mathrm{T} 0$ detector needed to be $<80 \mathrm{ps}$.

\section{The module design}

Figure 3 shows the schematic structure of the T0 detector, which consists of eight inner and eight outer MRPCs [15$18]$. In this design, a time resolution $<80 \mathrm{ps}$ and an efficiency $>95 \%$ (The efficiency is defined as the ration between the number of fired events and triggers.) are sought for the CEET0. In the following, we describe the efforts to build and test a T0 detector prototype, the details of the configuration of 


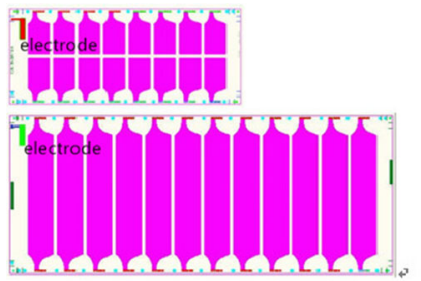

(a)

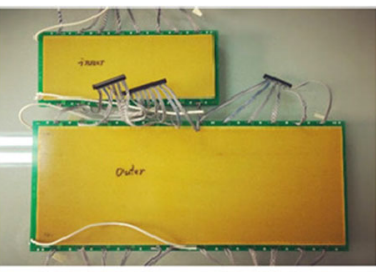

(b)

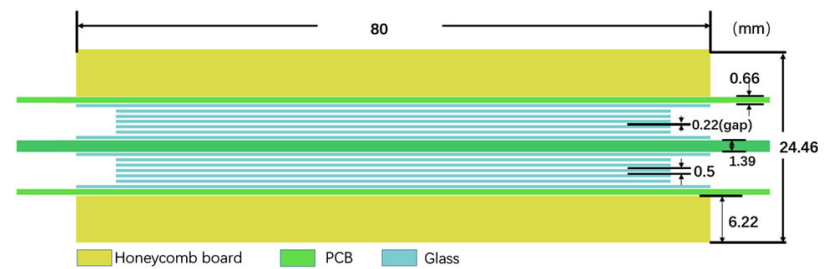

(c)

Fig. 4 MRPC module design and prototypes. The readout pad design for the inner layer is shown in the upper image (a) at the top left, and the design for outer layer of the MRPC is shown in the lower image (a) at the top left. A photograph of the finished MRPC prototypes is shown at the top right (b). The side view of the MRPC is shown in the bottom panel (c)

MRPCs, the readout electronics, the hadron and heavy ion beam test, and the results obtained from the beam test.

\subsection{The configuration of MRPC}

The inner and outer layers of the T0 detector are composed of eight MRPCs, which are suitable for high precision timing and fast triggering. Figure 4 shows the designs of the two kinds of MRPCs, for the inner and outer layers respectively. The smaller inner MRPCs shown at the top of Fig. 4a contain 16 single-end differential readout pads, each pad $3.05 \mathrm{~cm}$ long and $2.15 \mathrm{~cm}$ wide with a $0.35 \mathrm{~cm}$ gap, and the strips of the larger outer MRPCs are each $12.0 \mathrm{~cm}$ long, $2.6 \mathrm{~cm}$ wide and segmented by $0.4 \mathrm{~cm}$ gap, with a total of 12 dual-end differential readouts. The sensitive volume of the detector consists of $0.5 \mathrm{~mm}$ thick float glass plates consisting of a double-stack structure that is mirrored with respect to the central electrode [Fig. 4 bottom (c)] with twelve gas gaps. High voltages (HV) are applied to the external electrodes' surfaces. Each gap is supported by a nylon fishing line, with a diameter of $0.22 \mathrm{~mm}$. Figure $4 \mathrm{~b}$ shows a photograph of the finished inner and outer MRPC modules.

\subsection{Readout electronics}

The front-end electronics (FEEs) are located outside the gas box containing the MRPC module (Fig. 5a, which make use of the NINO chip [19]. This ultra-fast and low-power frontend amplifier/discriminator application specific integrated circuit (ASIC) was specially designed for the MRPC by the

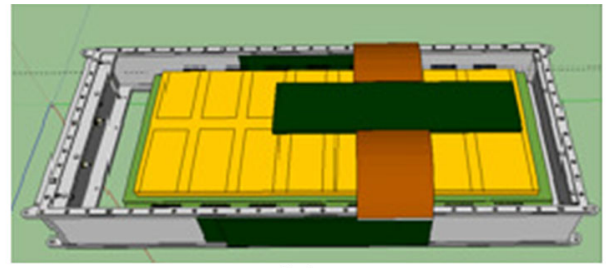

(a)

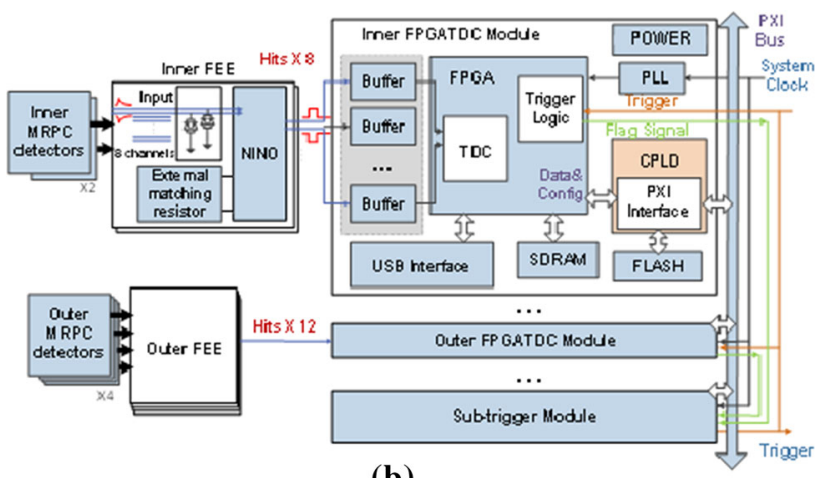

(b)

Fig. 5 Block diagram of the readout electronics and CSR T0 module

ALICE-TOF group. Figure $6 \mathrm{a}, \mathrm{b}$ shows the FEE boards. For the inner MRPC, which has 16 readout channels, two NINO chips are used. While for the outer MRPC, four chips are used to handle 24 channels. The off-chip resistor in the FEE (the "External matching resistor" in Fig. 5a is used for impedance matching [20], Each FEE module outputs corresponding LVDS signals with fast leading edges for timing purposes and the signal charge information is contained in its width. The signals from FEE are then processed by the field programmable gate array-based time-to-digital convertor (TDC) module [21]. The field programmable gate array (FPGA) TDC can achieve both leading and trailing edge time measurement in a single channel based on the carry chain structure within the FPGA slice resource with a time jitter of FPGA TDC $<25$ ps RMS for the leading edge. Trigger preprocessing, trigger matching based on Content-Addressed Memory (CAM) and Double Port Random Access Memory (DPRAM), and other functions are also integrated in one single FPGA device. The TDC module is designed based on PCI extensions for Instrumentation (PXI)-6U standard. The hardware configuration, data transfer, and online reconfiguration of the FPGA logic can be conducted by using a single board computer (SBC) located in Slot 0 through a PXI bus. A USB interface is also employed for system debugging. The block diagram of the readout electronics is shown Fig. 5b. Figure 6 shows photographs of the FEE and FPGA TDC modules.

After the readout electronics being designed and tested [22], preliminary commissioning tests with the four T0MRPC prototypes, including the two inner and two outer modules, were conducted in the laboratory with cosmic rays. 

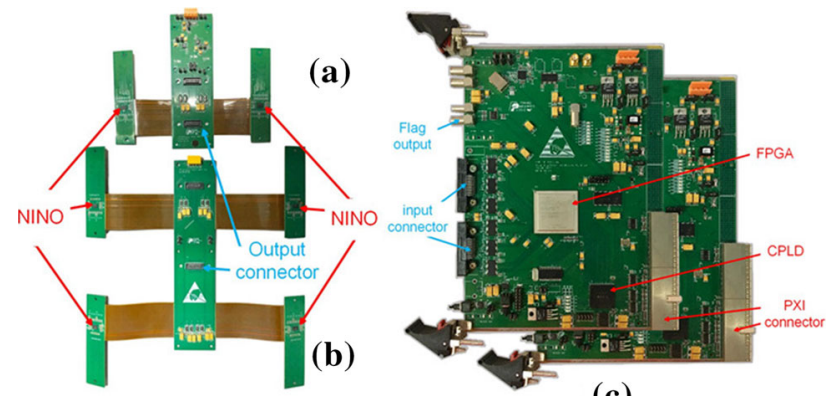

(c)

Fig. 6 NINO-based FEE modules for the inner (a) and outer (b) MRPC module. FPGA-based time-to-digital convertor is shown on the right (c). This FPGA TDC can achieve both leading and trailing edge time measurement with high precision. Trigger matching and other functions are also integrated

Then, the whole system was fully tested with hadron and heavy-ion beams to study its performance in detail.

\section{Hadron in-beam test}

\subsection{IHEP-E3 beam line at BEPC II facility}

The IHEP-E3 beam line at the Beijing Electron-Positron Collider II (BEPCII), Institute of High-Energy Physics (IHEP), Beijing, China has been used to study the characteristics of the CEE-T0 MRPCs and the functionality of the new electronics. Secondary particles (mainly protons, $\pi^{-/+}$and $e^{-/+}$) from an incident electron beam hitting a carbon target [23] were filtered and delivered to the IHEP-E3 line. Among these secondary particles, protons and pions were dominant. The particle momenta were tuned to $650 \mathrm{MeV} / \mathrm{c}$. A Cherenkov detector $(\mathrm{C} 0)$ and two scintillators ( $\mathrm{SC} 1$ and SC2 with an overlapping area of $5 \mathrm{~cm} \times 5 \mathrm{~cm}$ ) assembled by the XP2020 photomultiplier tubes were used for basic triggering. A coincidence of the two scintillators and an anticoincidence with the Cherenkov detector allowed the selection of protons and pions [24]. Three multi-wire chambers (MWPCs) were installed for the measurement of the beam trajectory. However, in this work, the Cherenkov detector and MWPC detectors were not included in the beam test.

\subsection{Test setup}

A sketch of the in-beam test setup is shown at the top of Fig. 7. The $\mathrm{SC} 1$ and $\mathrm{SC} 2$ scintillators provide the coincidence trigger, and the four small single-end readout scintillators $\left(2 \times 5 \mathrm{~cm}^{2}, \mathrm{BC} 420\right.$, divided into two groups: T1/T2 and T3/T4) coupled with fast photomultiplier tubes (PMT H6533) provide the accurate event reference time. ${ }^{1}$ The inner

$\overline{\left.\operatorname{Tr} 0=\frac{(T 1+T}{2}+T 3+T 4\right)}$. and outer MRPC modules for CSR-T0 detector were placed together with several other MRPC prototypes, ${ }^{2}$ at the downstream position of the test beam. T1 and T2 were placed at the upstream position, and $\mathrm{T} 3$ and $\mathrm{T} 4$ were at the downstream position relative to the CBM-TOF MRPC modules. If a particle passes through $\mathrm{SC} 1, \mathrm{SC} 2, \mathrm{~T} 1, \mathrm{~T} 2, \mathrm{~T} 3, \mathrm{~T} 4$, its signals are fed to the splitters. One copy is sent to the HPTDC (High Performance Time to Digital Convertor) for precise timing measurement $[19,26]$, while another output copy is fed to the discriminator. The HPTDC module, which has discrimination and signal transfer ability, was built by the USTC Electronics Group. After discrimination, the coincident signals of the $\mathrm{SC} 1$ and $\mathrm{SC} 2$ act as the trigger for the system. The digital signal from $\mathrm{T} 1$ to $\mathrm{T} 4$ act as the reference time start signals of the test system. The MRPC signals are amplified and discriminated by the FEE and then recorded by the FPGA-TDC. The difference between leading- and trailing-edge timing gives the signal width [time-over-threshold, (TOT)] information. The MRPC modules were placed in a gas-tight aluminum box and flushed with a working gas mixture of $90 \%$ R134a, $5 \%$ iso-butane, and $5 \%$ sulfur hexafluoride $\left(\mathrm{SF}_{6}\right)$. A photograph of the experimental setup is shown at the bottom of Fig. 7. The operational parameter values were set according to the cosmic ray test results $[27,28]$. In October 2016, two inner and two outer MRPCs for the CEE-T0 detector were tested at the IHEP-E3 line. The HV was set at $\pm 7200 \mathrm{~V}$ (The $\mathrm{HV}$ for the MRPC was set to $14.4 \mathrm{kV}$ ), and the threshold was set to $220 \mathrm{mV}$ (i.e., $36 \mathrm{fC}$ at the input of the NINO ASIC) during the test. The analysis of the collected data and the results are described in the following.

\subsection{Result of the in-beam test}

\subsubsection{Time resolution}

The IHEP-E3 beam was generated by bombarding with a primary electron beam a target such as $\mathrm{Cu}, \mathrm{Be}$ or $\mathrm{C}$. The secondary particles mix with $\mathrm{e}, \pi$ and $\mathrm{p}$, primarily protons and pions. So the first step of the analysis was to distinguish different particles. Figure 8 shows the Time-of-flight TOF $=\frac{(T 1+T 2)}{2}-\frac{(T 3+T 4)}{2}$ of protons and pions between the two scintillator sets of $\operatorname{Tr} 0$ detector. At a beam momentum of $650 \mathrm{MeV} / \mathrm{c}$ (The momentum of the beam was lower than the value in Ref. [23], because during the beam test, E3-line is preparing for maintenance.), protons deposit more energy in the $\operatorname{Tr} 0$ detector (scintillator) because of the larger $\mathrm{dE} / \mathrm{dx}$, and they travel more slowly than pions, resulting in larger

\footnotetext{
2 The prototype is CBM-TOF MRPC3b, and the detail information in the Ref. [25]. In the beam test, the DAQ system is independent and the data analysis is also independent.
} 


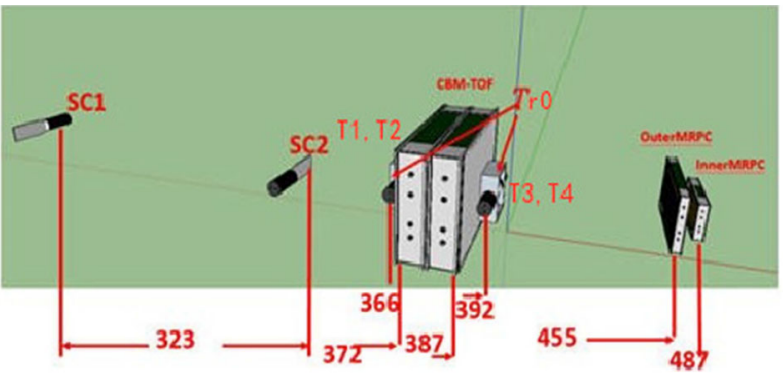

(a)

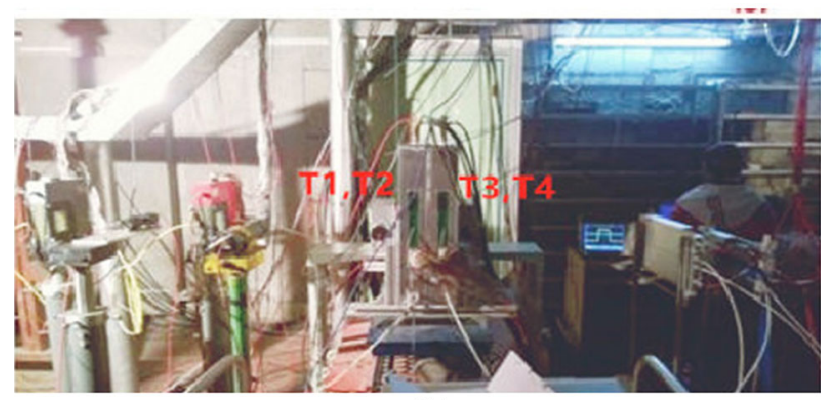

(b)

Fig. 7 Sketch (top a) and photograph (bottom b) of experimental setup at the E3 line of BEPCII. The numbers in the upper plot mark the position (in $\mathrm{cm}$ ) along the beam line

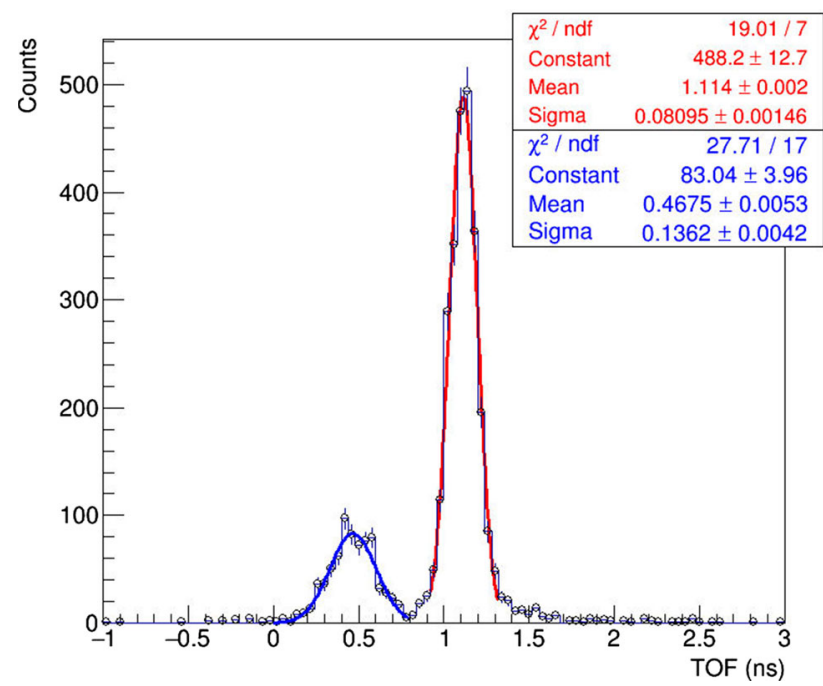

Fig. 8 The distribution of time of flight, TOF $=\frac{(T 1+T 2-T 3-T 4)}{2}$ The peak fitted with blue line are pions and the other read peak are protons

TOF values across a defined path length. ${ }^{3}$ However when the time resolution of the MRPC under test is evaluated, the average time of the scintillators $\left(\operatorname{Tr} 0=\frac{(T 1+T 2+T 3+T 4)}{4}\right)$ will be used as the reference time. Assuming that the time jitter of each scinitillator is the same then the time jitter

\footnotetext{
3 The distance between the T1/T2 pair and the T3/T4 pair is $\sim 26 \mathrm{~cm}$, corresponding to $T O F=\frac{L}{c p}\left(\sqrt{p^{2}+m_{\text {proton }}^{2} c^{2}}-\sqrt{p^{2}+m_{\pi}^{2} c^{2}}\right)=$ 640 ps.
}

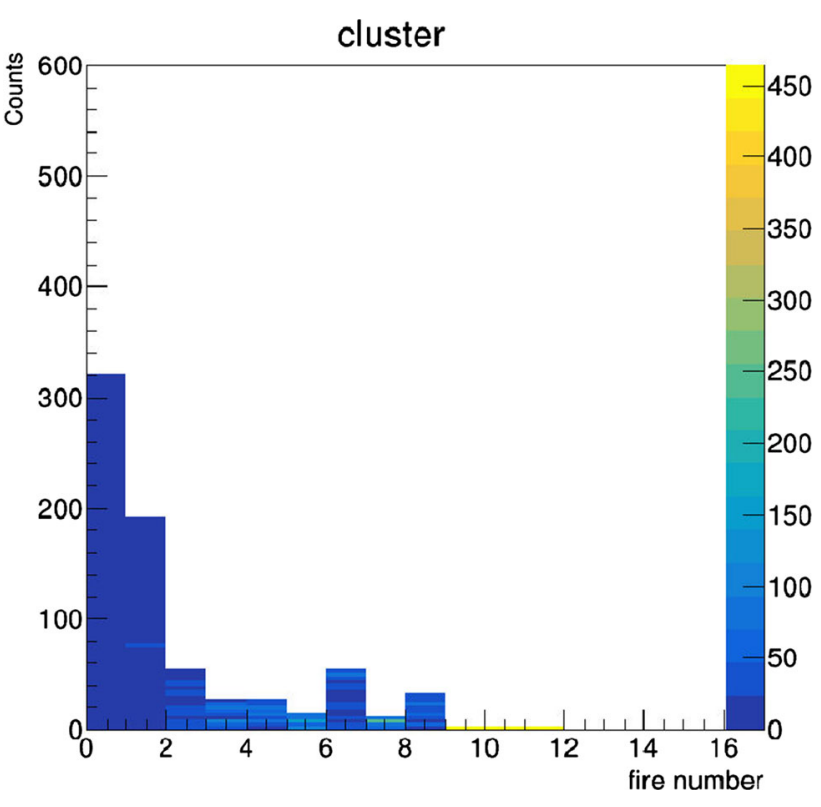

Fig. 9 Clustersize of the MRPC in hardon in-beam test

of $\operatorname{Tr} 0$ is half that of TOF $\left(\sigma_{T r 0}=\frac{\sigma_{T O F}}{2}\right)$; this gives the time jitter of $\operatorname{Tr} 0$ as $40 \mathrm{ps} / 60$ ps for protons and pions respectively.

The digital timing of the inner and outer MRPCs were filtered by the $\mathrm{T} 1$ to $\mathrm{T} 4$ scintillators, requiring all of them to have signals. The average cluster size, which is defined as the number fo fired strips or pads for a triggered beam crossing, of the MRPC is 1.6, as shown in Fig. 9. The timing of the inner and outer MRPCs was corrected with respect to Tr0, mainly for the time-amplitude slewing effect. The signal amplitude was estimated by its width (TOT). We have developed a new slewing correction method to correct the relationship between time and amplitude that combines fitting and bin counting. The MRPC timing and TOT plot were divided into several parts. To do the slewing correction, function fitting was used for the parts with enough statistics and the bin-by-bin counting method was used for the other parts (Fig. 10). Compared to the method used in Ref. [29], this new method has a better correction effect, especially for the channels with poor statistics, where no suitable function can be used to fit the data while the bin-by-bin method stills works well. The calibration strategy was pad-by-pad or strip-bystrip, so for every single pad or strip, we have very poor statistics. Figures 12 and 13 show the distributions of typical MRPC timing relative to Tr0 for proton and pion beams. The plots show that the time resolution of MRPC were measured to be $\sim 160 \mathrm{ps}$ for protons and $\sim 85 \mathrm{ps}$ for pions. The time resolutions of inner and outer MRPCs were found to be similar. The Fig. 11 shows the efficiency plateau at BEPCII. 


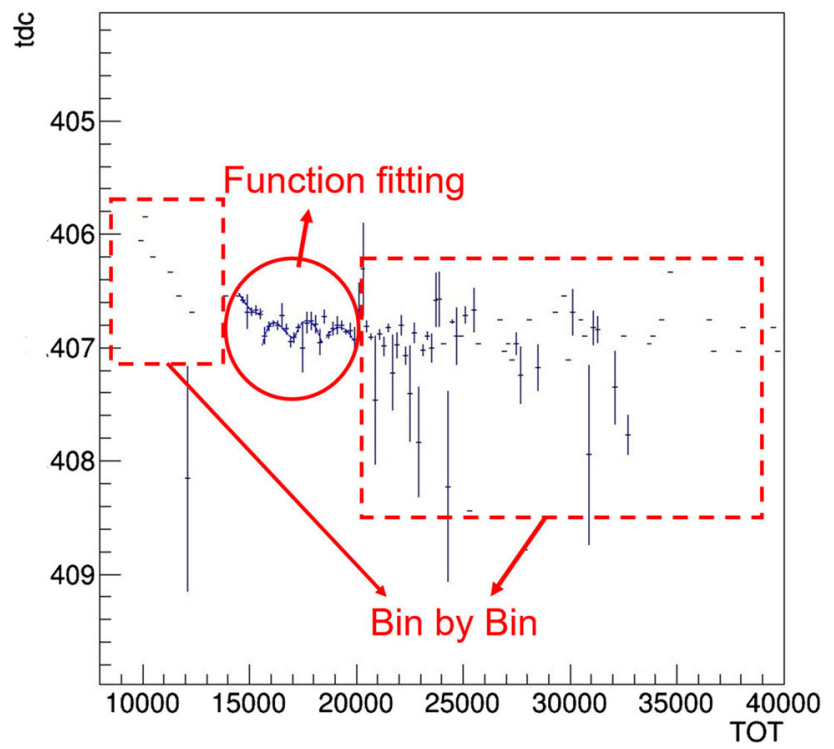

Fig. 10 MRPC timing and TOT plot divided into several parts. The circled part uses the function fitting and the rectangle parts use a binby-bin method to do slewing

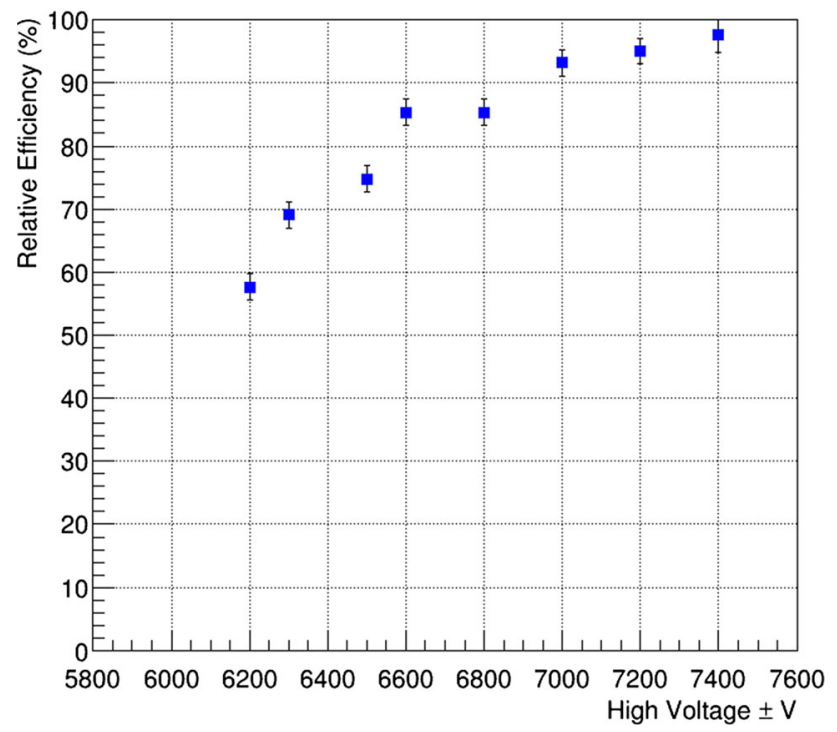

Fig. 11 Efficiency plateau at BEPCII

\subsubsection{Simulation}

Compared to the beam test result of the MRPCs with similar structure [16,17,27-30], the time resolution obtained from the simulations was significantly worse. The reason for this discrepancy is that, for beam test described in Ref. [29], the MRPCs were located in the middle position of the $\operatorname{Tr} 0$ detector. Thus the formula $\operatorname{Tr} 0=\frac{(T 1+T 2+T 3+T 4)}{4}$ provides a good estimation of the reference time for the MRPCs. However, in this work, the inner and outer MRPCs were placed downstream of the beam line at distances of $\sim 80$ and $\sim 100 \mathrm{~cm}$, respectively, from the geometric centre of the

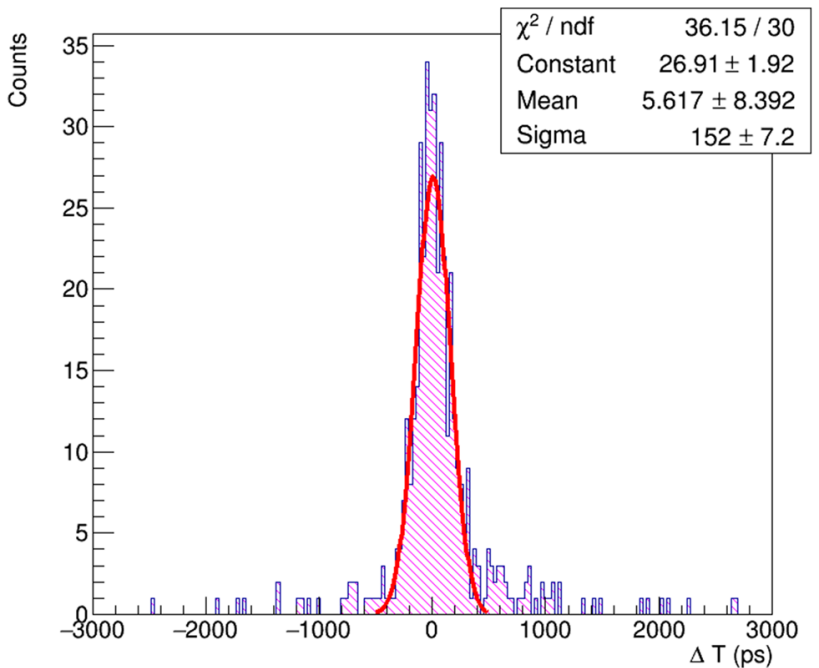

Fig. 12 MRPC time distribution relative to $T_{0}$ for a proton beam

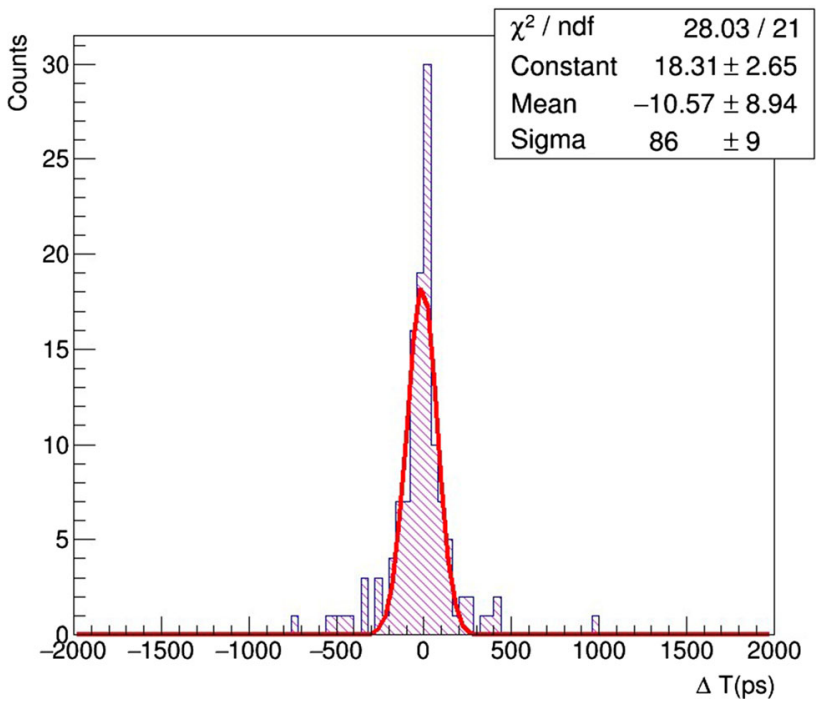

Fig. 13 MRPC time distribution relative to $\mathrm{T}_{0}$ for a pion beam

$\operatorname{Tr} 0$ detector. Because of the beam momentum uncertainty and the energy loss, multi-scattering etc. from the interaction with the detector materials, there was additional timing jitter compared to the measurement in Ref. [29]. This means what we measured and reported in Figs. 12 and 13 are actually $\Delta T=T_{M R P C}-\left(\operatorname{Tr} 0+T_{F}\right)$, where $\mathrm{T}_{F}$ is the time of flight between the $\operatorname{Tr} 0$ and CSR-T0 MRPC modules. $\mathrm{T}_{F}$ varies because of beam momentum variation, so the intrinsic MRPC time resolution with this effect taken into account should be $\sigma_{M R P C}=\sqrt{\sigma_{\Delta T}^{2}-\sigma_{T r 0}^{2}-\sigma_{T_{F}}^{2}}$.

To quantitatively understand the experimental results, we used the GEANT4 toolkit [31] to simulate the beam test experiment at IHEP-E3. The beam test system was simplified in the simulation by only considering the most relevant detectors, including the two thin slices of plastic scintilla- 
tor ( $\mathrm{SC} 1$ and $\mathrm{SC} 2,5 \mathrm{~cm} \times 5 \mathrm{~cm} \times 0.5 \mathrm{~cm}$ ) used for beam trigger, two groups of plastic scintillator strips (T1/T2 and $\mathrm{T} 3 / \mathrm{T} 4,5 \mathrm{~cm} \times 2 \mathrm{~cm} \times 1 \mathrm{~cm}$ ) used as the Tr0 detector, four CBM-TOF MRPC modules, and two CSR-T0 MRPC modules arranged along the beam direction (See Fig. 7). As much as possible, we used the materials same as the actual situation in the Geant 4 description. Along the beam direction, each MRPC module mainly includes the following materials: an aluminum gas-tight shielding box $(2-4 \mathrm{~mm}$ thick in total), three pieces of PCB (3-6 mm thick in total), 12 pieces of glass plate (6 mm thick in total), and a gas sensitive region (2.5 $\mathrm{mm}$ thick in total).

The beam momentum resolution was measured to be $\sim 2.5 \%$ at IHEP-E3 at an injection hadron beam momentum of $650 \mathrm{MeV} / \mathrm{c}$. These parameters were considered in the GEANT4 simulation. The energy loss and the multiple Coulomb scattering were also taken into account. The reference time $\operatorname{Tr} 0$, was the the average of all four channels of the Tr0 detector. An intrinsic MRPC timing uncertainty of $40 \mathrm{ps}$ and a $\operatorname{Tr} 0$ timing uncertainty of 20 ps were smeared into the simulation data. Figure 14 shows the distribution of $\mathrm{T}_{M R P C}-\mathrm{Tr} 0$. By comparing Fig. 14 to Fig. 12, we see that the simulation and experimental results are consistent with each other, indicating that the MRPC time resolution is $40 \mathrm{ps}$. Note that the Tr0 time jitter is smaller in the simulation (20 ps) than that shown in experiment ( 40 ps, Fig. 8 ), which includes an additional contribution from the beam momentum uncertainty as well as the intrinsic $\operatorname{Tr} 0$ uncertainty.

The above comparison was done for a proton beam. Because the IHEP-E3 beam also contains a small fraction of pions, we also compared the GEANT4 simulation results to the experimental MRPC time response for pions. We found that with an MRPC intrinsic timing uncertainty of 50ps and a Tr0 intrinsic timing uncertainty of $40 \mathrm{ps}$ smeared into the simulation, the simulated distribution of $\mathrm{T}_{M R P C}-\operatorname{Tr} 0$ (shown in Fig. 15) is consistent with the experimental results. The long tails on the time spectra are caused by the Coulomb multiply scattering and $\mathrm{dE} / \mathrm{dx}$ effects of the beam, which are more significant for the protons than for the pions.

Through these analyses of simulation and experiment data, we conclude that the time resolution was $\sim 40$ ps for proton and $\sim 50$ ps for pion at $650 \mathrm{MeV} / \mathrm{c}$, for both inner and outer MRPC modules. These values are also consistent with the results from previous test with similar MRPC structure. [29] (proton $\sim 41 \mathrm{ps,} \mathrm{pion} \sim 53 \mathrm{ps}$ ).

\section{Heavy-ion in beam test}

\subsection{Experimental setup}

In November 2016, the prototype of the T0 detector was tested with the heavy-ion beam at CSR. Figure 16 shows a

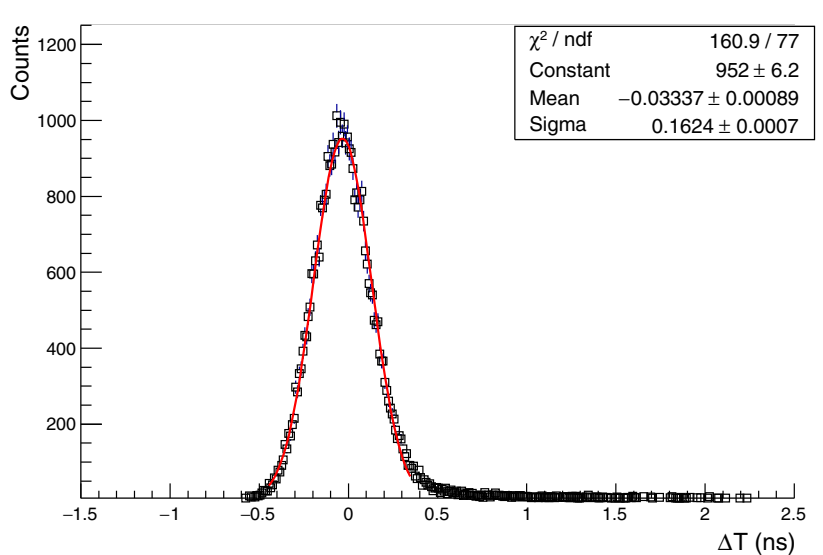

Fig. 14 Simulated distribution of $\mathrm{T}_{M R P C}-\mathrm{Tr} 0$ with proton beam. The beam momentum uncertainty and $\operatorname{Tr} 0$ timing jitter are included

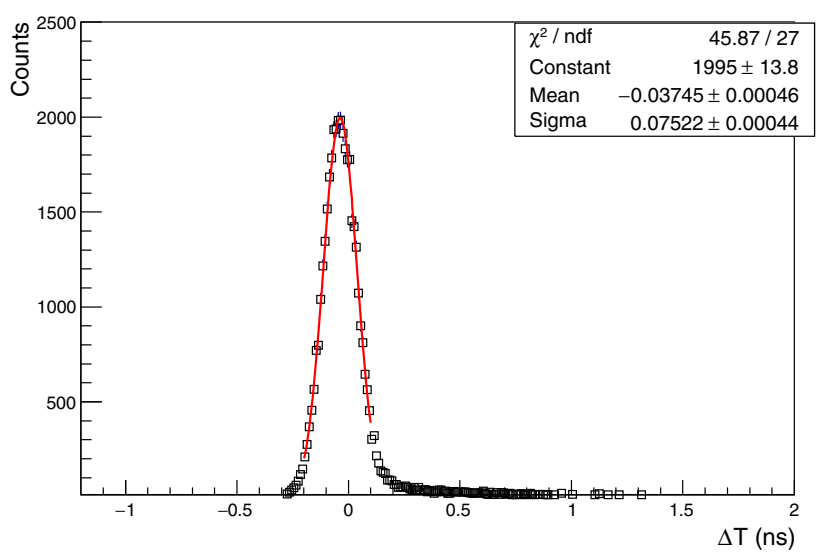

Fig. 15 Simulated distribution of $\mathrm{T}_{M R P C}-\mathrm{Tr} 0$ with pion beam. The beam momentum uncertainty and $\operatorname{Tr} 0$ timing jitter are included

sketch of the T0 detector test setup for the CSR externaltarget experiment. Only $1 / 4$ of the full T0 detector was built and tested, including two inner and two outer MRPCs. A photograph of the MRPC modules and mechanical structure is also shown in Fig. 16.

An Ar-40 beam with a kinetic energy of $300 \mathrm{AMeV}$ bombarded a lead and carbon target that was located at the geometrical centre of the $\mathrm{T} 0$ detector. Operated in a stand-alone mode, the T0 system was self-triggered by requiring that all four MRPCs were fired. The MRPC signal, after amplification and discrimination by the FEE, was sent to the digitalization electronics via a long cable $(\sim 10 \mathrm{~m})$. The total number of the readout channels was 80 , but we did not have enough electronics for this. Therefore, we used two kinds of TDCs with 40 channels were recorded by FPGA TDC modules and the other 40 channels were processed by the previously designed time digitization modules based on HPTDCs. Synchronization between these two types of TDC modules was achieved based on two techniques. First, a $40 \mathrm{MHz}$ system clock was fed to all the TDC modules, and thus the coarse 


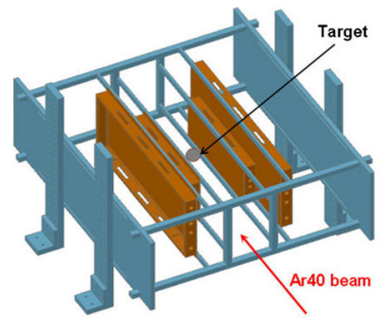

(a)

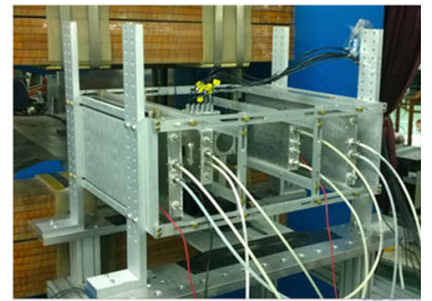

(b)
Fig. 16 Design of the T0 detector structure for the beam test at CSR (a) and a photograph of the experiment (b)

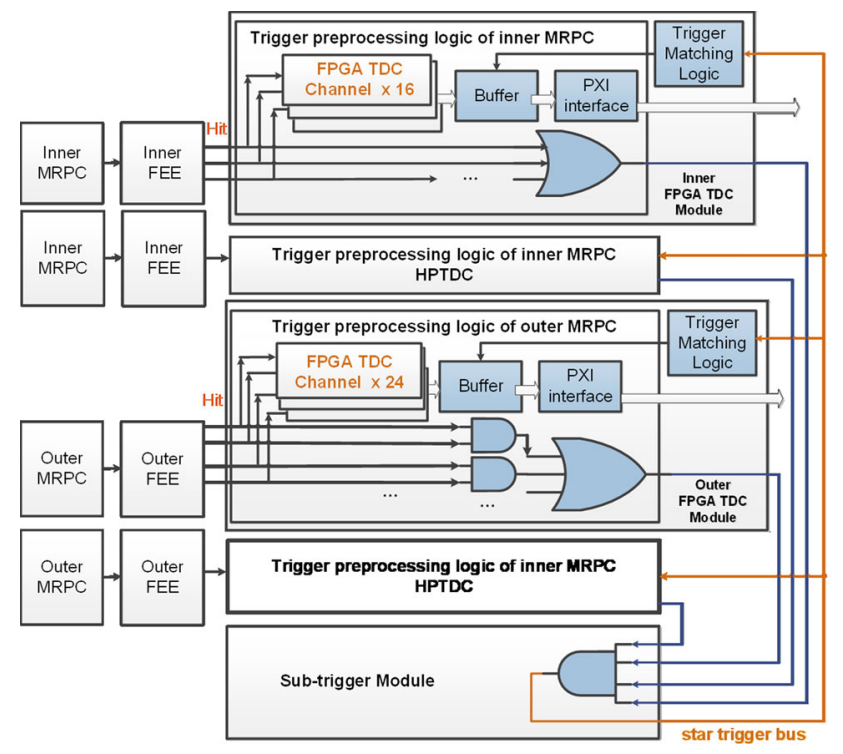

Fig. 17 Trigger processing and data readout

time and fine time (after interpolation) were all synchronized with the phase of this clock signal. Second, after the electronics were powered up, a global reset signal was generated, and it was fanned out from the sub-trigger module to all the TDC modules to clear the coarse time counter value and align the "start" points for the time measurement.

Figure 17 shows that the data from FPGA TDCs were stored in the internal buffers inside the FPGA, and the valid data were read out when a trigger signal was received. Trigger processing was organized in two hierarchies. The trigger mode in this experiment was as follows. In the first step, for the inner MRPC, the hit signals were fed to an logic OR gate. For the outer MRPC, the hit signals from the two ends of one MPRC strip were input to an logic AND gate and then further processed by the following logic OR gate. The above processing functions were implemented in the TDC modules. Next, the flag signals from both the inner and outer MRPC electronics modules were sent to the sub-trigger module and processed by its logic AND gate. Finally, a trigger signal was generated and transmitted to all TDC modules through the star trigger bus in the PXI crate.

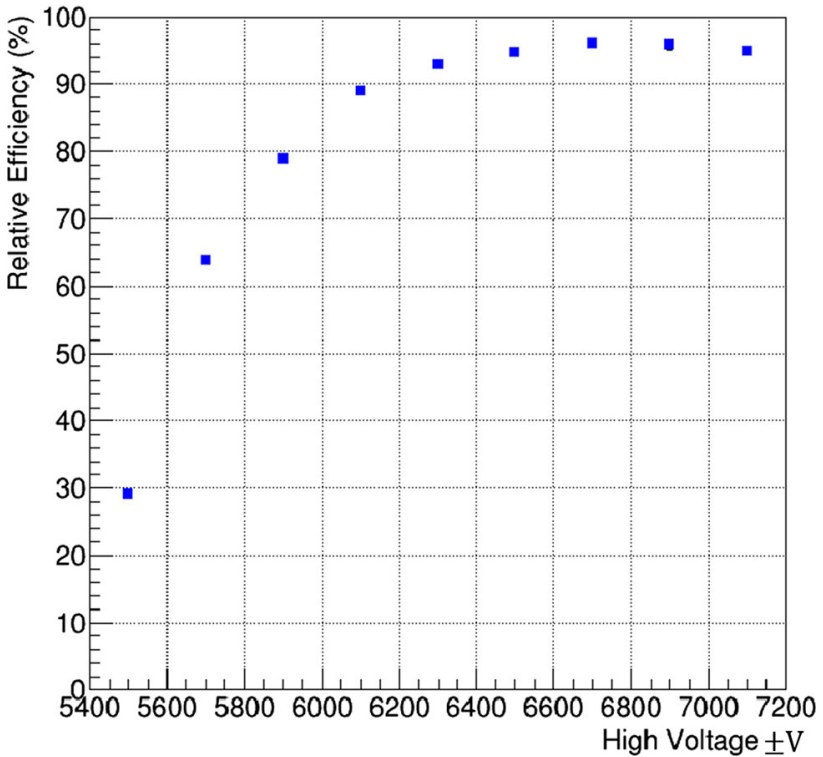

Fig. 18 MRPC detection efficiency vs. HV at CSR [28]

\subsection{Heavy-ion in-beam test}

\subsubsection{HV scan}

According to the rule $\mathrm{V}=V_{a} \frac{P_{0} T}{P T_{0}}$ [32], where $V_{a}$ is the applied voltage, $T$ represents the operating temperature and $P$ denotes the gas pressure, the operating voltage $\mathrm{V}$ changes with $\mathrm{P}$, and thus with altitude. Therefore, The operation condition of MRPC must adapt to the change of altitude in Lanzhou area $(\sim 1500 \mathrm{~m}$ a.s.l,with a normal atmosphere pressure of $5 / 6$ bar). We set up a cosmic-ray test during the beam time at CSR and checked the detection efficiency as a function of the applied HV, See Fig. 18 [28]. The efficiency was a relative one without any correction for the acceptance of the cosmic-ray, but one can clearly see a plateau. The working $\mathrm{HV}$ was chosen to be $6800 \mathrm{~V}$, which is significantly lower than the normal HV $(\sim 7200 \mathrm{~V})$ for the tests at the IHEP-E3 line (Fig. 11) and in the laboratory.

\subsubsection{Calibration procedure}

During the beam test the system was self-triggered, and there was no reference time and tracking information for the T0 detector. The event vertex position and field map that electronically channel match with the pad or strip number were also lacking. Therefore, the basic calibration strategy was to perform a relative correction to each channel. The time offset, TOT slewing correction, and particle velocity correction all needed to be calibrated.

The first step was to tune the time offset of each channel by comparing signals from neighbouring pads fired by a single particle. Each pair of inner and outer MRPCs of the T0 detec- 

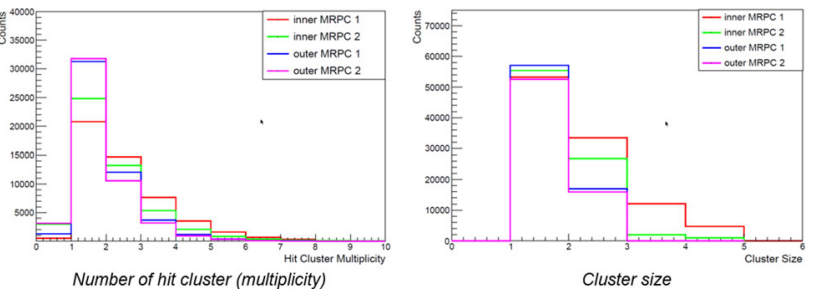

Fig. 19 Number of hit cluster and cluster size of the MRPC at beam time

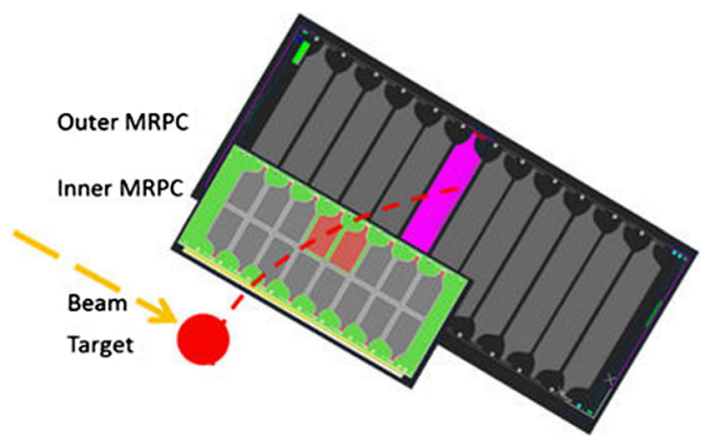

Fig. 20 Event selection: two neighbouring pads of inner MRPC and one strip of the outer MRPC were fired by a single particle

tor was combined to form one group (two groups in total in our test), and each group was calibrated separately. Figure 19 shows the cluster size and hit multiplicity plots. The maximum cluster size of the inner MRPCs (pad readout) was four, while for outer MRPCs (strip readout) was two. To suppress background hits, one fired strip on outer MRPC and two fired pads on inner MRPC within each group were required when calibrating the inner MRPC module. Figure 20 shows that the selected particle first hits the inner MRPC and then hits the outer MRPC, firing two neighbouring pads, so the hit point should be near the boundary region between the pads. In this case, when a single particle passes, it causes two fired channels of the inner MRPC, so their hit time should be the same. If channel 0 is considered to be the reference, and if all channels are iterated, the relative time offset can be evaluated and calibrated by a simple time shift for each channel. The calibration procedure for the outer MRPC modules is similar. In this case, it is necessary for there to be only one fired channel on both inner and outer MRPCs.

The second step is to correct the time slewing effect for each channel. As in the step 1 calibration, a single charged particle was fired at both inner and outer MRPCs. Next, the time difference between neighbouring channels was plotted as a function of $\mathrm{TOT}_{i}$, where $\mathrm{TOT}_{i}$ is the measured signal width of the channel to be calibrated. In our analysis the neighbouring pads/strips were those directly sharing one boundary with the selected pad/strip. For the inner MRPCs, one pad had three neighbouring pads (two neighbours for pads at the ends), and for outer MRPCs, one strip had two neighbouring strips (one neighbour for strips at the ends). For the outer MRPCs, TOT $_{i}$ was the mean TOT measurements from both ends. A typical slewing effect is illustrated in Fig. 21. A table of bin-by-bin centre value, rather than a fit curve, was used to correct the TOT dependence. The procedure was repeated until convergence was observed.

The next step in the calibration concerns the particle momentum spread. In the beam test, the momenta of the final state charged particles varied significantly from $\sim 200$ to $600 \mathrm{MeV} / \mathrm{c}$. Particles with momenta $<200 \mathrm{MeV} / \mathrm{c}$ are likely to be absorbed or scattered by the detector materials. The particle speed can be estimated by the time difference between the inner and outer MRPCs in the same group and their distance, $\mathrm{v}=\frac{L_{O u t}-L_{I n}}{T_{O u t}-T_{I n}}$, where $L_{O u t}, L_{I n}, T_{O u t}$ and $T_{I n}$ are the flight lengths and times from the collision point to the outer and inner MRPCs. For this test $L_{O u t}=22.5 \mathrm{~cm}$ and $L_{I n}=12.5 \mathrm{~cm}$. The event start time, T0, can be calculated by

$T_{0}=\frac{T_{\text {In }} L_{O u t}-T_{\text {Out }} L_{\text {In }}}{L_{\text {Out }}-L_{\text {In }}}$

This formula provides accurate collision time if there is no energy loss and if no multiple scattering effects are involved. However, at CSR energy, these factors cannot be ignored. Because relevant timing measurement from both groups of the MRPCs was necessary, to do a velocity calibration, we needed a reference $\operatorname{Tr} 0$. This was done by requiring each of the two groups of MRPCs to contain at least one valid track hitting both inner and outer MRPCs. Thus each group of MRPCs can give a measurement of Tr0, which can be used as a (relative) reference for the other group. The $T_{0}$ difference between the two groups was plotted vs. particle speed, as shown in Fig. 22 [28]. A clear velocity dependence was seen and used to calibrate the value of $T_{0}$.

There are some other factors that should be noted, such as the magnetic field and collision vertex uncertainty. The magnetic field was found to be $<0.1$ Tesla at the T0 detector location, so the bending radius of a proton was $>6.7 \mathrm{~m}$ if the momentum was required to be $>200 \mathrm{MeV} / \mathrm{c}$. Compared to the flight length $L_{O u t}$ and $L_{I n}$, the effect of magnetic field is small and, therefore, the effect was neglected in this analysis. The heavy-ion beam had a round shape and a root mean square (RMS) radius of $3 \mathrm{~mm}$. Because there was no measurement of the collision vertex position, this uncertainty also affected the $\mathrm{T} 0$ detector time resolution.

\subsubsection{Time resolution}

According to Eq. 1, the T0 detector's time resolution is mainly determined by the MRPC timing accuracy, the particle flight length, and momentum spread. The vertex uncertainty also affects the resolution by changing the flight length. 


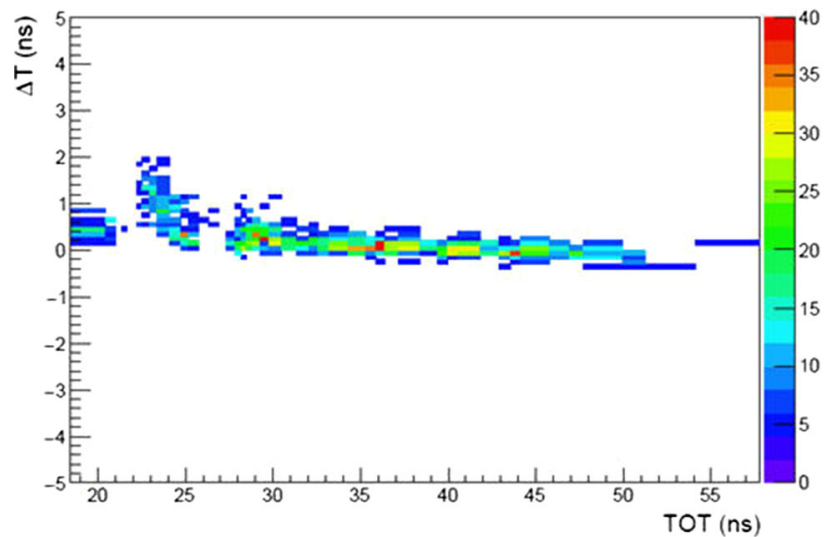

Fig. 21 Typical MRPC time vs TOT slewing correlation

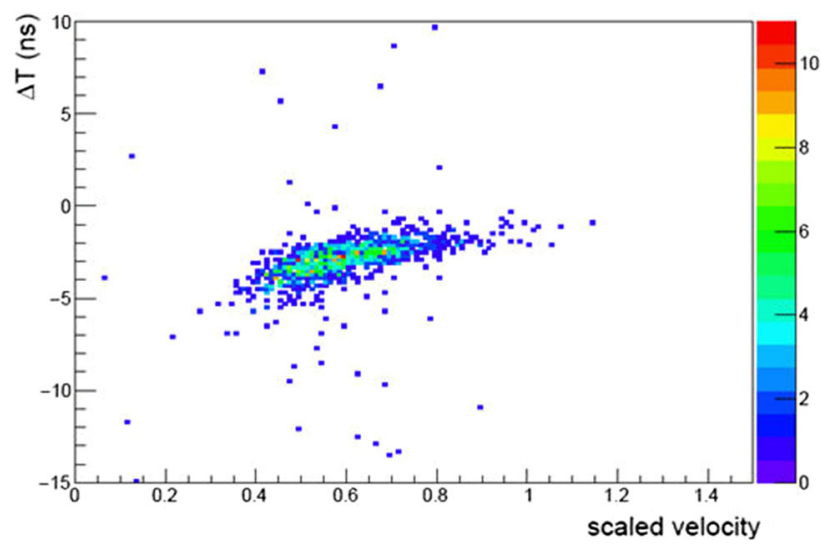

Fig. 22 Typical start time difference between two T0 groups vs. effective particle velocity

To estimate the timing performance of the $\mathrm{T} 0$ detector, the start time differences between the two T0 groups are calculated using $\Delta T_{T 0}=\frac{T_{01}-T_{02}}{2}$. Figure 23 (top a) shows their distribution after all corrections were applied. In this plot, each group was required to be hit by only one track, so $\sigma_{\Delta_{T 0}}$ represents a good measure of the $\mathrm{T} 0$ detector resolution, $\sigma_{T 0}$, by assuming $T_{0}=\frac{T_{01}+T_{02}}{2}$. When there were two tracks recorded by the $\mathrm{T} 0$ detector(one track for each group), the $\mathrm{T} 0$ time resolution was found by double-Gaussian fitting to be $\sim 100$ ps. See Fig. 23 (top a). We further studied the response uniformity of the T0 detector. Each group of the detector was divided into five regions according to the hit position along the beam direction. A total ten regions were scanned, and the time resolution was measured in a similar way to what was done to generate Fig. 23. The result is shown in Fig. 24 (bottom b). It's clear that a fairly uniform performance of the MRPCs was achieved.

Besides MRPC timing uncertainty, the observed T0 time resolution of $\sim 100$ ps (Fig. 23a, b) included contributions mainly from the collision vertex uncertainty, which was measured to be $\sigma_{V T X}=3 \mathrm{~mm}$ in the plane perpendicular to the beam direction. To study this contribution to the uncer-

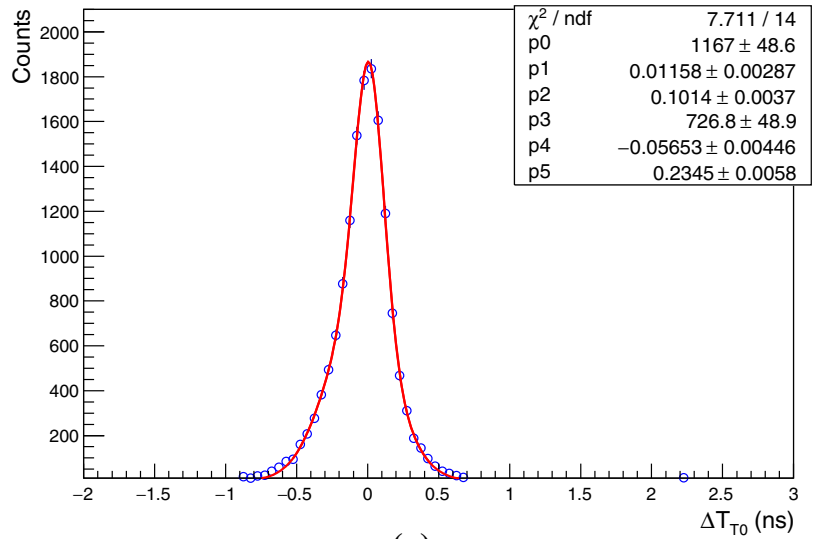

(a)

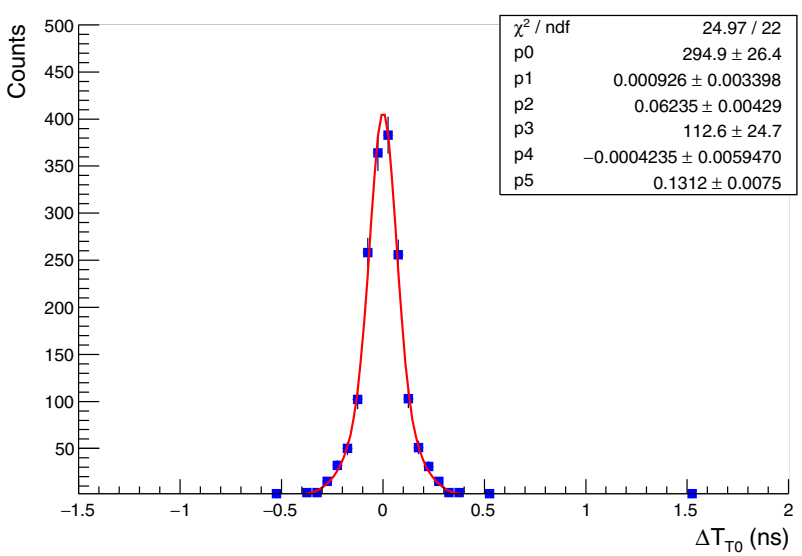

(b)

Fig. 23 Start time difference between two T0 groups (top), and within one T0 group (bottom)

tainty, events with two or more tracks hitting one of the two groups were selected, and the $\Delta T_{T 0}$ distribution was drawn for each pair of tracks. Because both tracks were from the same group, the effect of the vertex position variation largely cancelled out. With a double-Gaussian fit, the T0 time resolution of $\sim 60 \mathrm{ps}$ was determined. It is worthy noted that, in proposed CEE operation, the collision vertex will be precisely measured by other detectors, so it should not contribute to $\mathrm{T} 0$ time resolution. Both $\mathrm{Ar}+\mathrm{C}$ and $\mathrm{Ar}+\mathrm{Pb}$ collision data are analyzed. The results were found to be very similar and consistent with each other, despite some differences in hit multiplicity.

\subsubsection{Simulation study}

Owing to the lack of reference information of the collision time and other properties of the final state particles, we used an MC simulation to model the experimental result and provide a performance expectation for the $\mathrm{T} 0$ detector. The GEANT4 toolkit was used for the description of the detector and its response to particles generated by heavy-ion reac- 


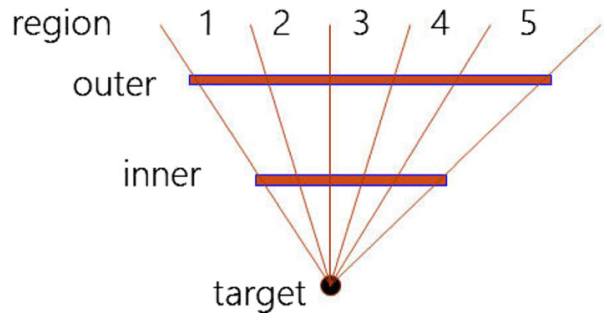

(a)

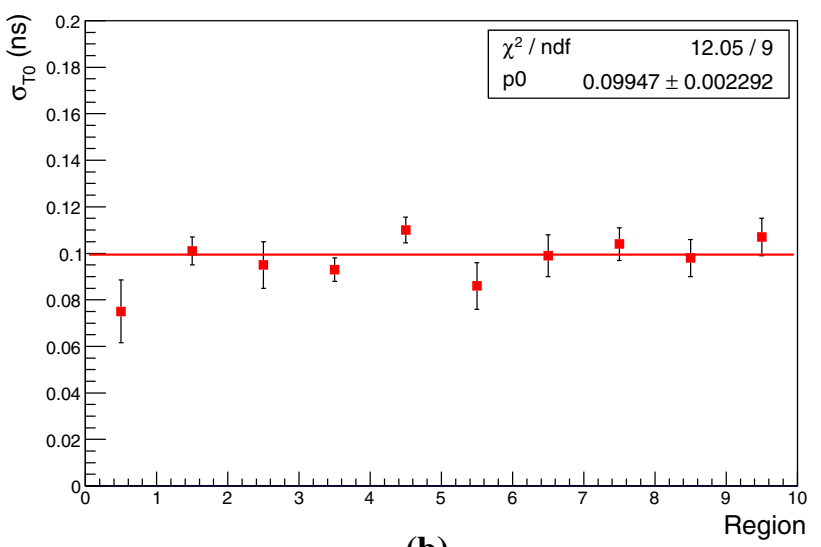

(b)

Fig. 24 (Top) Different hit position regions along the beam direction. (Bottom) T0 time resolution in each region

tion. Only the MRPCs, along with the boxes and FEE (the brown parts in Fig. 16) were included in the GEANT4 simulation. The target and other supporting structure were ignored. To improve the simulation efficiency, the T0 detector was assumed to have full acceptance, like in Fig. 3. The heavy-ion collision event was simulated by the UrQMD3.4 generator [14]. The incident beam consisted of argon- 40 nuclei and the target was carbon-12. The kinetic energy of the beam was 300 $\mathrm{AMeV}$. We have assumed 50,75, and $100 \mathrm{ps}$ timing resolution for the MRPCs in the simulation. It was found that the simulation fit the experimental result best with a timing smearing of 50 ps. See Fig. 25. The top (a) plot shows the deduced $T_{0}$ time resolution by a single track, while the bottom (b) plot illustrates its dependence on the number of tracks that hit the T0 detector. The collision vertex was fixed, so its position did not contribute to the overall resolution. By comparing the $T_{0}$ time resolution with two tracks in Fig. 25 (bottom b) and in Fig. 23 (bottom b), the MRPC time resolution, including contributions from electronics, particle momentum variation and magnetic field, should be $<50 \mathrm{ps}$. This is consistent with the results for IHEP-E3 beam test and validated the excellent performance of the T0 prototype, which completely fulfils the design goal.

Figure 25 (bottom $\mathrm{b}$ ) also shows that the $\mathrm{T} 0$ time resolution quickly decreased to $\sim 50 \mathrm{ps}$ for more than five track, and it was saturated at $\sim 30 \mathrm{ps}$. We would expect even better perfor-

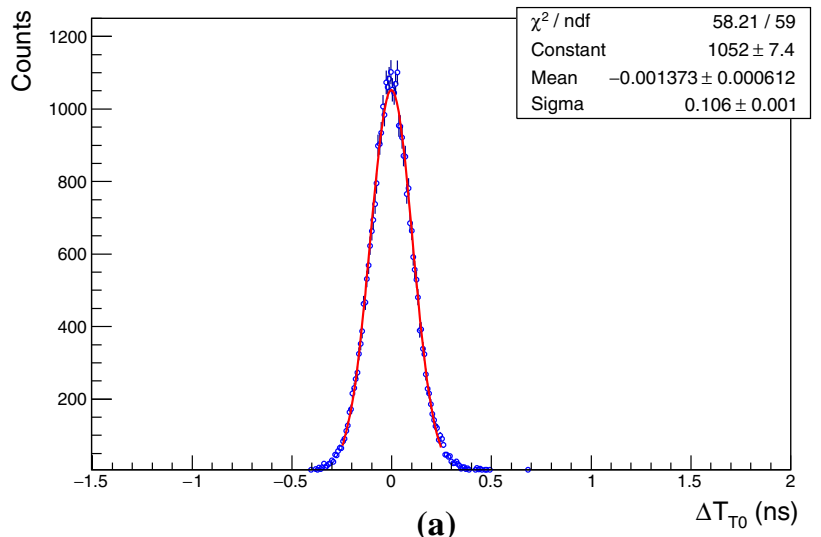

(a)

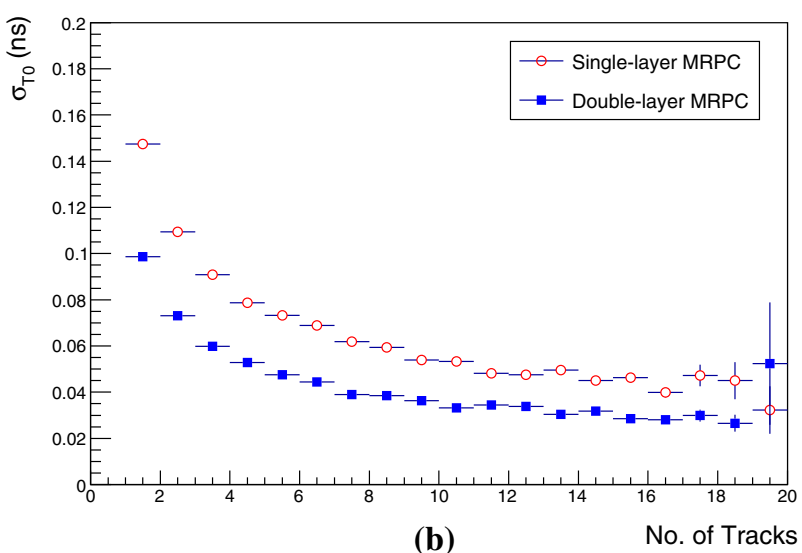

Fig. 25 T0 time resolution from simulation data

mance by fine-tuning and calibration of the experiment. For comparison, the $\mathrm{T} 0$ time resolution with a one-layer design for the T0 detector is also shown, it is $\sim 30-50 \%$ worse than the double-layer design. This confirmed our expectation when designing this detector.

\section{Conclusions}

Based on MRPC technology, a prototype CSR-T0 detector (of 1/4 acceptance) was designed and produced. The MRPCs, FEE and the timing performance of the T0 prototype have been tested with a hadron beam and a heavy-ion beam. The efficiency of a single MRPC module was also examined, but the overall trigger efficiency for a T0 detector could not be quantified owing to the lack of beam counting rate information. A GEANT4-based simulation was done to evaluate the experimental data analysis results. The intrinsic time resolution of an MRPC, including electronics' contribution, was found to be $<50 \mathrm{ps}$ for charged hadrons at a momentum of $<1 \mathrm{GeV} / \mathrm{c}$. From the heavy-ion beam test at CSR, the timing performance of the $\mathrm{T} 0$ prototype has been evaluated and met 
our expectation, which suggests that our high expectations for a full-coverage $\mathrm{T} 0$ are promising.

However, the evaluation of the efficiency of the T0 detector has a real problem, because we do not have record of the beam-target reaction rate, so we have no point of reference. We can only estimate the efficiency from a simulation and the efficiency of a single MRPC. One thing we may do is to find the global time dependence of the T0 trigger and compare it to the beam luminosity to ascertain whether their time structure are the same ( $\sim 2$-s beam spill per $30 \mathrm{~s})$.

Acknowledgements We thank the E3 test beam group, IHEP, Beijing and the CSR beam group, Institute of Modern Physics (IMP), Lanzhou for their support to conduct the beam tests successfully. This project is supported by the National Natural Science Foundation of China (U1232206), the International Cooperation and Exchanges Project of NSFC (11420101004), the National Program on Key Basic Research Project of China-973 Program (2015CB856902), and the CAS Center for Excellence in Particle Physics (CCEPP).

Data Availability Statement This manuscript has associated data in a data repository. [Authors' comment: All data included in this manuscript are available upon request by contacting with the corresponding author and all original pictures can be obtained through the corresponding author.]

Open Access This article is licensed under a Creative Commons Attribution 4.0 International License, which permits use, sharing, adaptation, distribution and reproduction in any medium or format, as long as you give appropriate credit to the original author(s) and the source, provide a link to the Creative Commons licence, and indicate if changes were made. The images or other third party material in this article are included in the article's Creative Commons licence, unless indicated otherwise in a credit line to the material. If material is not included in the article's Creative Commons licence and your intended use is not permitted by statutory regulation or exceeds the permitted use, you will need to obtain permission directly from the copyright holder. To view a copy of this licence, visit http://creativecomm ons.org/licenses/by/4.0/.

Funded by SCOAP ${ }^{3}$.

\section{References}

1. W. Myers, W. Światecki, Nuclear equation of state. Phys. Rev. C 57(6), 3020 (1998)

2. P. Braun-Munzinger, J. Stachel, The quest for the quark-gluon plasma. Nature 448(7151), 302 (2007)

3. S. Zhang, J. Chen, H. Crawford, D. Keane, Y. Ma, Z. Xu, Searching for on-450set of deconfinement via hypernuclei and baryonstrangeness correlations. Phys. Lett. B 684(4-5), 224-227 (2010)

4. L. Mc Lerran, RHIC physics: the Quark gluon plasma and th condensate: Four lectures, 2003. arXiv:hep-ph/0311028

5. L. McLerran, Quarkyonic matter and the revised phase diagram of QCD. Nuclear Phys. A 830(1-4), 709c-712c (2009)

6. A. Andronic, D. Blaschke, P. Braun-Munzinger, J. Cleymans, K. Fukushi-ma, L. McLerran, H. Oeschler, R. Pisarski, K. Redlich, C. Sasaki et al., Hadron production in ultra-relativistic nuclear collisions: quarkyonic matter and a triple point in the phase diagram of QCD. Nuclear Phys. A 837(1-2), 65-86 (2010)
7. Z.-Q. Feng, G.-M. Jin, Probing high-density behavior of symmetry energy from pion emission in heavy-ion collisions. Phys. Lett. B 683(2-3), 140-144 (2010)

8. W.-J. Xie, J. Su, L. Zhu, F.-S. Zhang, Symmetry energy and pion production in the Boltzmann-Langevin approach. Phys. Lett. B 718(4-5), 1510-1514 (2013)

9. K. Sumiyoshi, H. Toki, Relativistic equation of state of nuclear matter for the supernova explosion and the birth of neutron stars. Astrophys. J. 422, 700-718 (1994)

10. A.W. Steiner, M. Prakash, J.M. Lattimer, P.J. Ellis, Isospin asymmetry in nuclei and neutron stars. Phys. Rep. 411(6), 325-375 (2005)

11. Z.-G. Xiao, G.-C. Yong, L.-W. Chen, B.-A. Li, M. Zhang, G.-Q. Xiao, N. Xu, Probing nuclear symmetry energy at high densities using pion, kaon, eta and photon productions in heavy-ion collisions. Eur. Phys. J. A 50(2), 37 (2014)

12. Z. Xiao, L.-W. Chen, F. Fu, B.-A. Li, G. Jin, H. Xu, G. Yong, M. Zhang, Nuclear matter at a HIRFL-CSR energy regime. J. Phys. G Nuclear Particle Phys. 36(6), 064040 (2009)

13. L. Lü, H. Yi, Z. Xiao, M. Shao, S. Zhang, G. Xiao, N. Xu, Conceptual design of the HIRFL-CSR external-target experiment. Sci. China Phys. Mech. Astron. 60(1), 012021 (2016). https://doi.org/ 10.1007/s11433-016-0342-x

14. UrQMD, Urqmd. http://urqmd.org/ (2005)

15. V. Gapienko, O. Gavrishchuk, A. Golovin, A. Semak, S.Y. Sychkov, Y.M. Sviridov, E. Usenko, M. Ukhanov, Studying the counting rate capability of a glass multigap resistive plate chamber at an increased operating temperature. Instrum. Exp. Tech. 56(3), 265-270 (2013)

16. S. Yong-Jie, Y. Shuai, L. Cheng, Q. Sen, X. Lai-Lin, F. Zai-Wei, H. Yue-Kun, C. Hong-Fang, T. Ze-Bo, S. Ming et al., A prototype mrpc beam test for the BESIII ETOF upgrade. Chin. Phys. C 36(5), 429 (2012)

17. E.C. Zeballos, I. Crotty, D. Hatzifotiadou, J.L. Valverde, S. Neupane, M. Williams, A. Zichichi, A new type of resistive plate chamber: the multi-gap RPC. Nuclear Instrum. Methods Phys. Res. Sect. A Accelerat. Spectrom. Detect. Associat. Equip. 374(1), 132-135 (1996)

18. M. Spegel, A. Collaboration et al., Recent progress on rpcs for the alice tof system. Nuclear Instrum. Methods Phys. Res. Sect. A Accelerat. Spectrom. Detect. Associat. Equip. 453(1-2), 308-314 (2000)

19. F. Anghinolfi, P. Jarron, F. Krummenacher, E. Usenko, M. Williams, Nino, an ultra-fast, low-power, front-end amplifier discriminator for the time-of- flight detector in Alice experiment. In: IEEE Nuclear Science Symposium. Conference Record (IEEE Cat. No. 03CH37515), Vol. 1. IEEE 2003, pp. 375-379 (2003)

20. F. Anghinolfi, P. Jarron, A. Martemiyanov, E. Usenko, H. Wenninger, M. Williams, A. Zichichi, Nino: an ultra-fast and low-power front-end amplifier/discriminator asic designed for the multigap resistive plate chamber. Nuclear Instrum. Methods Phys. Res. Sect. A Accelerat. Spectrom. Detect. Associat. Equip. 533(1-2), 183187 (2004)

21. J. Wang, S. Liu, L. Zhao, X. Hu, Q. An, The 10-ps multitime measurements averaging TDC implemented in an FPGA. IEEE Trans. Nuclear Sci. 58(4), 2011-2018 (2011)

22. P. Deng, L. Zhao, J. Lu, P. Xia, J. Liu, M. Li, S. Liu, Q. An, Readout electronics of t0 detector in the external target experiment of CSR in HIRFL. IEEE Trans. Nuclear Sci. 65(6), 1315-1323 (2018)

23. L. Jia-Cai, W. Yuan-Ming et al., A test beam upgrade based on the BEPC-LINAC. High Energy Phs. Nuclear Phys. 28(12), 1269 (2004)

24. BESIII Collaboration, et al., The construction of the BESIII experiment. Nuclear Instrum. Methods Phys. Res. Sect. A Accelerat. Spectrom. Detect. Associat. Equip. 598(1), 7-11 (2009)

25. D. Hu, X. Wang, Y. Sun et al., Beam test of CBM-TOF MRPC prototype. J. Instrument. 14(09), C09014 (2019) 
26. J. Christiansen, HPTDC high performance time to digital converter, CERN/EP-MIC, version 2.1, CERN, Geneva (2002). http://cds. cern.ch/record/1067476

27. M. Shao, X. Dong, Z. Tang, Y. Xu, M. Huang, C. Li, H. Chen, Y. Lu, Y. Zhang, Upgrade of the calibration procedure for a star time-of-flight detector with new electronics. Meas. Sci. Technol. 20(2), 025102 (2008)

28. D. Hu, M. Shao, Y. Sun, C. Li, H. Chen, Z. Tang, Y. Zhang, J. Zhou, H. Zeng, X. Zhao et al., A t0/trigger detector for the external target experiment at csr. J. Instrument. 12(06), C06010 (2017)

29. S. Yang, Y. Sun, C. Li, Y. Heng, S. Qian, H. Chen, T. Chen, H. Dai, H. Fan, S. Liu et al., Test of high time resolution mrpe with different readout modes for the besiii upgrade. Nuclear Instrum. Methods Phys. Res. Sect. A Accelerat. Spectrom. Detect. Associat. Equip. 763, 190-196 (2014)
30. A. Akindinov, A. Alici, F. Anselmo, P. Antonioli, M. Basile, G.C. Romeo, L. Cifarelli, F. Cindolo, A. De Caro, S. De Pasquale et al., Results from a large sample of mrpc-strip prototypes for the alice tof detector. Nuclear Instrum. Methods Phys. Res. Sect. A Accelerat. Spectrom. Detect. Associat. Equip. 532(3), 611-621 (2004)

31. S. Agostinelli, J. Allison, K.A. Amako, J. Apostolakis, H. Araujo, P. Arce, M. Asai, D. Axen, S. Banerjee, G. Barrand et al., Geant4a simulation toolkit. Nuclear Instrum. Methods Phys. Res. Sect. A Accelerat. Spectrom. Detect. Associat. Equip. 506(3), 250-303 (2003)

32. D. Gonzalez-Diaz, D. Belver, A. Blanco, R.F. Marques, P. Fonte, J. Garzín, L. Lopes, A. Mangiarotti, J. Marín, The effect of temperature on the rate capability of glass timing rpcs. Nuclear Instrum. Methods Phys. Res. Sect. A Accelerat. Spectrom. Detect. Associat. Equip. 555(1-2), 72-79 (2005) 